\title{
Applicability of the ReproQ Client Experiences Questionnaire for Quality Improvement in Maternity Care
}

Marisja Scheerhagen, Henk F Van Stel, Dominique D.J.C. Tholhuijsen, Erwin E Birnie, Arie Franx, Gouke J. Bonsel

Background. The ReproQuestionnaire measures the client's experience with maternity care, following the WHO responsiveness model. In 2015, the ReproQ was appointed as national client experience questionnaire and will be added to the national list of indicators in maternity care. For using the ReproQ in quality improvement, the questionnaire should be able to identify best and worst practices. To achieve this, ReproQ should be reliable and able to identify relevant differences. Methods and findings. We sent questionnaires to 17,867 women 6 weeks after labor (response 32\%). Additionally we invited 915 women for the retest (response 29\%). Next we determined the test-retest reliability, the Minimally Important Difference (MID) and six known group comparisons, using two scorings methods: the percentage women with at least one negative experience and the mean score. The reliability for the percentage negative experience and mean score was both 'good' (Absolute agreement $=79 \%$; intraclass correlation coefficient $=0.78$ ). The MID was $11 \%$ for the percentage negative and 0.15 for the mean score. Application of the MIDs revealed relevant differences in women's experience with regard to professional continuity, setting continuity and having travel time. Conclusions. The measurement characteristics of the ReproQ support its use in quality improvement cycle. Test-retest reliability was good, and the observed minimal important difference allows for discrimination of good and poor performers, also at the level of specific features of performance. 
1 Title: Applicability of the ReproQ Client Experiences Questionnaire for Quality Improvement in

2 Maternity Care

3

4 Authors: Marisja Scheerhagen, $\mathrm{MSc}^{1,2}$; Henk F van Stel, $\mathrm{PhD}^{3}$; Dominique JC Tholhuijsen,

5 BSc ${ }^{4}$; Erwin Birnie, $\mathrm{PhD}^{2}$; Arie Franx, $\mathrm{MD}, \mathrm{PhD}^{5}$; Gouke J Bonsel, $\mathrm{MD}, \mathrm{PhD}^{1,2}$.

6

7 (1) Erasmus Medical Centre, Department of Obstetrics and Gynecology, Rotterdam, The

8 Netherlands

9 (2) University Medical Center Utrecht, Department of Obstetrics and Gynecology, Academic

10 Collaborative Maternity Care, Utrecht, The Netherlands.

11 (3) University Medical Center Utrecht, Julius Center for Health Sciences and Primary Care,

12 Department of Health Technology Assessment, Utrecht, The Netherlands

13 (4) Erasmus University Rotterdam, Institute of Health Policy and Management, Rotterdam, The

14 Netherlands.

15 (5) University Medical Center Utrecht, Department of Obstetrics and Gynecology, Utrecht, The

16 Netherlands

18 Corresponding author at: Henk van Stel PhD, Julius Center for Health Sciences and Primary

19 Care, Department of Health Technology Assessment, University Medical Centre Utrecht, 
20 Heidelberglaan 100, Room STR 5.117, 3584 CX Utrecht, The Netherlands. Telephone: +31 8875

21 59378. Fax: +31 8875 55482. E-mailadres: $\underline{\text { H.vanStel@umcutrecht.nl. }}$ 


\section{ABSTRACT}

24 Background. The ReproQuestionnaire measures the client's experience with maternity care,

25 following the WHO responsiveness model. In 2015, the ReproQ was appointed as national client experience questionnaire and will be added to the national list of indicators in maternity care. For using the ReproQ in quality improvement, the questionnaire should be able to identify best and worst practices. To achieve this, ReproQ should be reliable and able to identify relevant differences.

Methods and findings. We sent questionnaires to 17,867 women 6 weeks after labor (response $32 \%$ ). Additionally we invited 915 women for the retest (response $29 \%$ ). Next we determined the test-retest reliability, the Minimally Important Difference (MID) and six known group comparisons, using two scorings methods: the percentage women with at least one negative experience and the mean score. The reliability for the percentage negative experience and mean score was both 'good' (Absolute agreement $=79 \%$; intraclass correlation coefficient $=0.78$ ). The MID was $11 \%$ for the percentage negative and 0.15 for the mean score. Application of the MIDs revealed relevant differences in women's experience with regard to professional continuity, setting continuity and having travel time.

Conclusions. The measurement characteristics of the ReproQ support its use in quality improvement cycle. Test-retest reliability was good, and the observed minimal important 
41 difference allows for discrimination of good and poor performers, also at the level of specific

42 features of performance.

\section{INTRODUCTION}

44 Client experiences are considered to be important independent indicators for health care

45 performance (Valentine et al. 2007; Valentine et al. 2003). Being relevant for its own sake, client

46 experiences also affect clinical outcome through several pathways (Campbell et al. 2000; Sitzia

47 \& Wood 1997; Wensing et al. 1998; Williams 1994). For example, clients who truly understand

48 the explanation of their caregiver are more likely to comply to treatment or to change lifestyle,

49 and arguably a patient-unfriendly clinical staff or an intimidating hospital-setting will not support

50 recovery.

51 The routine measurement and use of client experiences play an indispensable role in systematic

52 quality improvement (Haugum et al. 2014; Weinick et al. 2014). For that purpose, the client

53 information can be used in a two-stage quality cycle. In the first stage, care providers that

54 perform above or below average are identified. This process is also called benchmarking

55 (Department of Health 2010; Ellis 2006; Ettorchi-Tardy et al. 2012; Kay 2007). In the second

56 stage assumed underperformers are invited to improve their results followed by an internal

57 quality cycle, where above-average performers ('best practices') may give guidance. Translated

58 technically, the quality cycle starts with quantification of individual client experiences and

59 clinical outcomes (casemix-adjusted), followed by the ranking across providers. Next, after 
60 defining thresholds, under- and best-performing units are defined. Finally, client experiences and

61 other outcomes are analyzed in more detail. Preferably this break down of data is combined with

62 face-to-face interactions among professionals. This more refined analysis offers tangible targets

63 for improvement, unlike the global outcomes used in benchmarking.

64 To include clients' experiences with maternity care in the routine care quality evaluation and

65 quality improvement, and in view of professionals, clinical organizations and health insurance

66 companies who urged for measuring quality from the perspective of the client, we developed the

67 Repro Questionnaire (ReproQ) (Scheerhagen et al. 2015b). This integral measure covers the

68 period from first antenatal intake up to the postpartum period. The ReproQ consists of 8 domains

69 (33 experiences items), following the so-called WHO Responsiveness model (Valentine et al.

70 2007; Valentine et al. 2003). All items strictly focus on service delivery from the clients'

71 perspective.

73 Previously we demonstrated the feasibility, internal consistency and construct validity of the

74 ReproQ (Scheerhagen et al. 2015b). In current paper we focus on psychometric properties

75 needed to assess the suitability of the ReproQ for the two-stage quality improvement process.

76 This suitability rests on two pillars: (1) are the judgments of pregnant women reliable, or stated

77 otherwise, if the client survey is repeated do we get the same average and the same ranking of

78 units? And (2) if we observe a quantitative difference between the average judgments of two care 
79 units - say 0.2 ReproQ points in our case - is this difference a relevant one? Epidemiologists

80 developed a robust method to decide which differences are relevant in case of difficult to grasp

81 clinical outcomes, the so-called minimal important difference (MID) approach. We tested these

82 properties of the ReproQ to establish whether the ReproQ is suitable for both global

83 benchmarking with a summary score (hence the assignment of below- and above-average

84 performance), and for detailed profiling of providers or units or client groups once the

85 underperforming units of client groups have been identified using the MID.

87 The data presented were collected during the provisional implementation of ReproQ measurement in about $1 / 3$ of all perinatal units (hospitals with nearby midwife practices) in the

89 Netherlands between October 2013 and January 2015. In 2015, the ReproQ was appointed as a national client experience questionnaire and will be added to the national list of indicators in

91 maternity care (CPZ 2015). Before the ReproQ was added to this national list, indicators only measured clinical outcomes (e.g. mortality, morbidity or complications) or parameters of

93 professional performance. Adding the ReproQ to this list of indicators meets the WHO's

94 recommendation to measure performance of health care systems also from the client's perspective. As indicator of performance the ReproQ should meet the conditions for a successful quality improvement cycle. This study explores two of these conditions: the ReproQ's reliability of the performance measurements and the MID as an aid to identify relevant differences between 
98 clients or perinatal units. The focus in this paper is on client's experiences with labor, because

99 this is the key-event in maternity care. Antenatal care aims to create the best possible situation

100 or starting point for labor. Antenatal risk assessment will be performed, and if necessary

101 preventive measures and treatment of these risks are embedded. Provision of postnatal care is

102 provided to reflect the outcome of the delivery for mother and child. Additionally, care during

103 delivery is comparable in most Western countries, while antenatal and postnatal care are subject

104 to more variation across countries or health systems. 


\section{MATERIALS \& METHODS}

\section{Repro Questionnaire}

108 The questionnaire consists of two analogous versions: version A covers the experiences during pregnancy (antenatal) and version B covers the experiences during birth and the postnatal period.

110 Version A is presented at about eight months gestational age, version B about six weeks postpartum. Each version asks for experiences at two instances, in case of version B postpartum experiences during labor, and experiences in the subsequent postpartum week respectively. As questions only differ with respect to the context referred to (say, experienced respect is asked for first antenatal visits, late in pregnancy, during labor, and during post-partum care), the resulting dataset represents a similar measurement covering four time intervals. In this article we focus on data from version $\mathrm{B}$ on the experiences during labor, the 3rd time point.

The 8-domain WHO responsiveness model is the conceptual basis of the ReproQ.

Responsiveness is the way a client is treated by the professional and the environment in which the client is treated. Responsiveness is operationalized as four domains represent interactions with health professionals (dignity, autonomy, confidentiality, and communication), and four domains that reflect experiences with the organizational setting (prompt attention, access to

123 family and community support, quality of basic amenities, and choice and continuity of care)

124 (see table 1) (Valentine et al. 2007; Valentine et al. 2003). The response mode of the experience 
125 items uniformly used four categories: "never", "sometimes”, “often", and "always", with a

126 numerical range of 1 (worst) to 4 (best). An additional question which two domains are

127 considered the most important, allows for a personalized scoring. Additional questions provide

128 information on 1) the rating of the global experience, 2) the process of care process, the location

129 of care (e.g. home or hospital) and the primary health professional being responsible (e.g.

130 midwife or obstetrician); 3) the clinical outcome of both mother and child, as perceived by the

131 mother; 4) information about previous pregnancies; and 5) client's socio-demographic

132 characteristics.

133 Content validity of the ReproQ-version-0 was determined through structured interviews with

134 pregnant women, women who recently had given birth, and health care professionals. All

135 Responsiveness domains were judged relevant. Construct validity of the adapted ReproQ-

136 version-1 was determined through a web-based survey, and based on response patterns;

137 exploratory factor analysis; association of the overall score with a Visual Analogue Scale; and

138 known group comparisons. The exploratory factor analysis supported the assumed domain

139 structure and suggested several adaptations. Correlation of the VAS rating and overall ReproQ

140 score supported validity for the antenatal and postnatal versions of the ReproQ. Further details

141 are described elsewhere (Scheerhagen et al. 2015b).

143 [Table 1. Description of the eight WHO Responsiveness domains] 


\section{Data collection}

147 In current study, data were obtained from three sources. The majority of data were collected by three postnatal care organizations (organizations that deliver postnatal care over a period of seven to 10 days). Additional data were collected by the national Birth Centre Study (a university-based research organization), and from 10 perinatal units (a hospital with associated midwifery practices). There were no exclusion criteria regarding organization, health care professional or client.

Data collection implied that clients were invited to participate by their care provider on behalf of the research team. With their consent, name and e-mail address were obtained and provided to the organization that distributed the digital survey. Women provided formal informed consent at the beginning of the questionnaire. For the Birth Centre Study and 10 perinatal units, the research team received client's name and e-mail information for recruitment after written informed consent had been obtained. The person who included the woman can, theoretically, be the same as the health care professional in charge of the delivery (usually gynecologist or community midwife), but this is highly unlikely to be the case and not typical of our obstetric care system. 
162 During data collection, an extensive data privacy protocol applied. The Medical Ethical Review

163 Board of the Erasmus Medical Center, Rotterdam, the Netherlands, approved the study protocol

164 (study number MEC-2013-455).

165

Data were collected in two waves. The first wave was between October 2013 and January 2015.

Six weeks after the expected date of labor, all participating women received an invitation to fill

out the postnatal ReproQ questionnaire. Non-responding women received a reminder two weeks

later. These data were used to determine the MID and compare the known groups. The second

wave occurred during October 2014 and January 2015. All women who previously filled out the

postnatal ReproQ measurement in the first wave were invited to fill out their experiences again

for the test-retest comparison. Excluded from invitation were women whose answers in the

postnatal ReproQ were largely incomplete. The intended test-retest interval was 14 days. Since

women's situation might change during the test-retest interval, we added the following item for verification. "Have you experienced something important in the last two weeks?"

\section{Participating women}

Sample size was not formally calculated since we had no prior data to use as input data.

Additionally, a formal sample size calculation seems questionable since statistical testing does 
181 national implementation of this survey would provide sufficient numbers of responses for the

182 study questions. For the MID and known groups comparison, we included all usable responses.

183 For the test-retest, we aimed at 200 usable questionnaires.

184 In the first wave, we invited 17,867 women who recently had given birth, of whom 5,760

185 responded to the survey (32\%). We excluded 877 women, because they filled out less than two

186 of the following characteristics: ethnicity, educational level, care process, and experienced

187 outcome of the mother and baby. We considered these background data as critical to describe the

188 study participants in sufficient detail, and to understand and interpret the ReproQ scores and the

189 associated MIDs. In the second wave, we invited 915 women for the retest, of whom 265

190 responded (29\%). We excluded 57 women for the retest, because their situation changed

191 negatively or was unknown. We did so because, a test-retest analysis requires that context and

192 conditions between the test and retest situations remain unaltered (De Vet et al. 2011). To judge

193 representativeness, we compared the characteristics of 208 women in the test-retest with the

1944675 women who filled out the test once using standard Chi square tests.

\section{ReproQ Score Model}

197 We used two scoring models to summarize women's experiences: the proportion women with

198 negative experience(s) (in short: 'percentage negative') and the mean score. Both were

199 calculated for the eight individual domains, the four personal domains, the four setting domains 
200 and a total score across all domains. Percentage negative was defined as filling out the response

201 category 'never' in at least one of the domains and/or filling out 'sometimes' in a domain that the

202 client identified as most important. The percentage negative method avoids compensation of a

203 negative experience by positive experiences on other items of domains, whereas the mean scores

204 allow the compensation of negative experiences. The mean scores were computed as unweighted

205 average-scores, treating never (1), sometimes (2), most of the time (3) and always (4)

206 numerically.

208 Data analysis

\section{Test-retest reliability}

210 Test-retest reliability was assessed using three measures. (1) For the percentage negative, we used the percentage absolute agreement, classified as excellent' (90\%-100\%), 'good' (75\%89\%), 'moderate' (60\%-74\%), or 'poor' $(<60 \%)$ (Singh et al., 2011). (2) For the mean scores, we used the Intraclass Correlation Coefficient (two way mixed model, absolute agreement, single

214 average), classified as: 'excellent' $(\geq .81)$, 'good' $(.61-.80)$, 'moderate' $(.41-.60)$, 'poor' $(\leq$

215 .40) (Singh et al. 2011). (3) Finally we created the Bland-Altman plot, calculating the bias (or

216 mean difference between test and retest scores) and the limits of agreement, equal to the mean

217 difference $\pm 2 * \mathrm{SD}$ of that mean difference (Bland \& Altman 1986; Streiner \& Norman 2008). 


\section{Minimally Important Difference}

220 We determined the MID using (1) the anchor-based (or the difference in score between two

221 adjacent levels of an anchor-question (Copay et al. 2007)) and (2) distribution-based method (or

222 the difference in distribution of observed scores (Revicki et al. 2008)), each having their merits.

223 As anchor-question we used the global rating of a client's experience: "Overall, how would you

224 rate the care received during your labor and care after birth?' (in short: 'Global rating'). This

225 anchor-question emerged as best option in a review study of the Picker Institute (Graham \&

226 Maccormick 2012). Women could respond to this question on a 10-point VAS. We determined

227 the mean score and the percentage negative of the individual domains, personal, setting and total

228 scores for the VAS ratings 7, 8 and 9. We used the global rating of ' 8 ' as reference category,

229 being the mode in our data (Copay et al. 2007). Next, the MID was calculated by subtracting

230 these mean scores of the adjacent categories 7 and 9 from the mean score of the reference, being

2318 , to check if the differences 7-8 and 8-9 were equal (Copay et al. 2007). The same procedure

232 was used to calculate the MID of the percentage negative. The distribution-based MID was only

233 calculated for the mean score. To determine the MID with distributed-based methods, we

234 calculated the standard error of measurement (SEM) (Wyrwich et al. 2002), and one half of the

235 standard deviation ( $1 / 2 \mathrm{SD})$ (King 2011; Norman et al. 2003). The SEM is estimated by the

236 baseline SD of the measurement multiplied by the square root of 1 minus its reliability

237 coefficient (ICC from the test-retest assessment) (Rejas et al. 2011; Vernon et al. 2010; Wyrwich 
238 et al. 2002). A difference larger than 1 SEM is thought to indicate a true difference between

239 groups (Copay et al. 2007; Revicki et al. 2008). The $1 \frac{1}{2} \mathrm{SD}$ margin is regarded as a relevant

240 difference as well (Copay et al. 2007; Norman et al. 2003; Revicki et al. 2008).

241

242

Clinical known-group comparison

243 We used six so-called known group comparisons (in terms of clinical outcome) to assess the

244 discriminative validity of the ReproQ. Here we determine if women from different 'known

245 groups' also have different mean experience scores and percentage negative (setting, personal,

246 overall), and if these differences exceed the anchor-based MIDs for 7-8 and 8-9.

247 We made the following 'known groups': First, we compared the scores of women who before the

248 labor did and did not meet the health care professional who supervised their labor, this being a

249 proxy of professional continuity (Saultz \& Lochner 2005). Second, for setting continuity, we

250 compared the scores of women who were entirely low risk versus women who shifted from low-

251 risk to high-risk during parturition. These women have the highest mortality and morbidity risk

252 (Evers et al. 2010; Poeran et al. 2015). Third, we compared the scores of women who started

253 their labor in office hours (8:00 am-5:00 pm, Mondays to Fridays) versus past office hours

254 (Gould et al. 2005; Gould et al. 2003; Stephansson et al. 2003; Urato et al. 2006). Fourth, we

255 compared the scores of women who had to travel 15 minutes or more with women who had to

256 travel less than 15 minutes. In agreement with literature, we only included women in this 
257 comparison who were transferred from home to hospital during parturition and whose birth was

258 unplanned.(Poeran et al. 2014; Ravelli et al. 2011). Fifth, we compared the scores of women who

259 had an emergency with women who had a planned caesarean section (Elvedi-Gasparovic et al.

260 2006). Finally, as proxy of concentration of care, we compared the scores of women who

261 delivered in small hospitals ( $<750$ labors annually (first quartile) vs. large hospitals $(\geq 1500$

262 labors annually (fourth quartile) (Finnstrom et al. 2006; Moster et al. 1999; Moster et al. 2001;

263 Phibbs et al. 1996; Tracy et al. 2006). 


\section{RESULTS}

265 Table 2 presents the characteristics of responding women who filled out the test $(\mathrm{n}=4675)$ and

266 women who filled out the retest $(n=208)$. Mean age was 31 years $(S D=4.3) ; 398(8 \%)$ women

267 were of non-Western background; and 368 (8\%) women reported to have a low educational level

268 (both percentages slightly below national average). About half of the women gave birth for the

269 first time (52\%; about national average), and 2313 (48\%) women did not know the health care

270 professional who supervised labor. 527 (11\%) women were referred to secondary care during

271 their pregnancy; $1724(36 \%)$ were referred during parturition (about the national average) and

$272618(12 \%)$ women had a cesarean section (below the national average of $18 \%$ ). The

273 characteristics of women who filled out the retest differed significantly in terms of ethnic

274 background (more Western women), setting continuity (more women were referred to secondary

275 care during pregnancy), and global rating (women gave a higher global rating).

276

277 [Table 2. Characteristics of women who filled out the test $(n=4675)$ and the retest $(n=208){ }^{\$}$.]

\section{Test-retest reliability}

280 Table 3 shows the test-retest reliability. All experience items combined, $47 \%$ of the women

281 reported one or more negative experiences filling out the test. When filling out the retest, $40 \%$ of women reported one or more negative experiences. The absolute test-retest agreement of 'having 
283 a negative experience' was 78.8\% (CI: 72.6\%-84.2\%). The ICC of the total scores

$284\left(\operatorname{mean}_{\text {test }}=3.79 ;\right.$ mean $\left._{\text {retest }}=3.78\right)$ was 0.78 , showing good reliability. The mean test-retest

285 difference of the total score was 0.01 ; limits of agreement were +0.31 and -0.31 ). The reliability

286 of the personal and setting scores was similar to the reliability of the total score.

287

288 The level of agreement regarding negative experiences within individual domains was excellent, except for the domains Autonomy and for Choice and Continuity that showed good agreement.

In these two domains, women also reported a higher level of negative experiences (Autonomy:

27.9\%; Choice and Continuity: $18.3 \%)$ than in other domains $(<7 \%)$. The ICCs varied between

moderate (0.49 for Confidentiality) and good ( 0.70 for Communication). The bias was minimal $(\leq 0.05)$ and was highest in the domains Dignity and Basic Amenities.

[Table 3. Test-retest reliability of the experience during labor, in terms of mean score and

percentage women with a negative experience $(n=208)$.]

\section{Minimally Important Difference}

Table 4 shows the MID results, using the two scoring models, including the results for the 7-8

and 8-9 differences. Using the percentage negative experience, the MID was $11.0 \%$, based on the difference between the global ratings of 7 and 8 . This means that the respondents rating their 
302 experiences compared to the respondents with the rating 8 . When comparing the rating of 8 with

3039 , the MID was $9.2 \%$. If we focus on the personal score, the MID using the 7-8 difference was

$3048.5 \%$, which was comparable to the MID of 8.9\% using the 8-9 difference. For the setting score,

305 the MID 7-8 was 5.4\%, which was smaller than the MID 8-9 (6.2\%). The MIDs of the individual

306 domains were all $\leq 8 \%$. Using the ReproQ overall mean instead of the percentage negative

307 experiences, the anchor-based MID based on the 7-8 distance was 0.15 ; when based on the 8-9

308 rating difference the MID was 0.10 . The mean-MIDs of the personal score were slightly larger

309 than the mean-MIDs of the setting score, and the domain MIDs showed some heterogeneity;

310 both patterns were also observed using MIDs for negative experiences.

311 The use of the mean score also allowed the computation of a distribution-based MID. The

312 distribution-based mean-MIDs of the 7-8 differences of the personal, setting and total score were

313 similar to the anchor-based MIDs. In case of the individual domains, all distribution-based mean-

314 MIDs were a somewhat larger than the anchor-based mean-MIDs.

316 [Table 4: Minimally important difference of the experience during labor based on the mean

317 scores and the percentage women that had a negative experience $(n=3841)]$ 
320 Figure 1A shows the impact of six known groups with an assumed influence on client

321 experiences, using the percentage of negative experiences as scoring model. Two out of six

322 comparisons showed differences in agreement with expectations. Already knowing the

323 professional who supervised labor (i.e. continuity of professional), had a considerable impact:

324 the differences in total score and personal score of women who knew and did not know their

325 professional were larger than the associated MIDs (7-8 difference). Similarly, referral during

326 labor (i.e. discontinuity of setting) was associated with differences in total, personal and setting

327 scores larger than the MID.

329 Figure 1B shows the same known groups comparison, now using the mean ReproQ scores and

330 the associated MID. The difference in mean overall, setting and personal scores between women

331 who received only primary care and women who were transferred during parturition was larger

332 than the corresponding MIDs (7-8 difference). All three differences scores of personal continuity

333 and setting continuity were larger than the MIDs (8-9 difference). Further details are presented in

334 supplementary file 1.

336 [Figure 1: Difference in ReproQ in terms of negative score (A) and mean score (B), between the

337 least preferred and the most preferred state, in 6 known-groups $(n=4883)]$ 
338

339

340

341

342

343

344

345

346

347

348

349

350

351

352

353

354

355

356

\section{DISCUSSION}

To determine the suitability of ReproQ in the two-stage quality improvement cycle, we assessed its test-retest reliability and determined the MID according to two methods. Test-retest reliability was good for both scoring models. The anchor-based MID of the percentage negative experiences was $11 \%$; the anchor-based MID of the mean score was 0.15 (on a range of $1-4$ ). The distribution-based MIDs (SEM) proved about similar to the anchor-based mean-MID of the overall, personal and setting scores. However, for the domain scores the SEM exceeded the anchor-based mean-MIDs. The known-group comparisons showed that knowing the professional that supervised your labor and not being referred during labor had considerable impact on the experiences scores. As the observed ReproQ scores deviated more than the MID, we believe this instrument can be used as a benchmark with an interpretation of meaningful differences beyond statistical significance. Thus, the ReproQ can successfully identify areas that need improvement in subgroups of clients. One should be aware that the MID cannot be used to identify changes in (poor) experiences within clients.

Applying the percentage negative on the test-retest reliability showed that the reliability of the domains was higher than for the summery scores. This is surprising, because the likelihood to report a negative experience in both the test and retest is considerably larger for the summary scores than for the domains. 
357 For the individual domains, fewer women reported a negative experience when filling out the

358 retest than the test. The domains Autonomy and Choice and Continuity showed similar

359 percentages of negative experiences in the test and retest, though the reliability of these domains

360 was low compared to the other domains. This indicates that women who reported a negative

361 experience filling out the test are not the same women that reported a negative experience filling

362 out the retest. Possible explanations for these effects are recall bias and/or response shifts, e.g.

363 women adjust their opinion due to sharing their experiences with family and friends.

364 The summary scores showed higher reliability than the domain scores when using the mean

365 score method. The explanation is that ICCs invariably increase when the summary scores include

366 more items. When calculating a summary score, differences within a domain can be

367 compensated by differences between the domains. This increases the stability of the summery

368 scores.

369 The reliability of the domains Confidentiality and Social considerations was somewhat lower

370 than for the other domains. It is possibly that women feel that these concepts are difficult to

371 judge, which increases the fluctuations in domain scores.

373 Both the negative-MID and the mean-MID varied across adjacent response categories. When the

374 global rating increases neither the percentage negative decreases nor the mean scores increase

375 linear in all scores. This suggests that a gain in client experience as the result of quality 
376 improvement is not similar to the loss in client experience as the result of deterioration. One

377 explanation is that clients do not weigh all domains are equally in the global rating. Another

378 explanation is that respondents are not inclined to use the extreme response categories.

379 The distribution-based MIDs (SEM) were similar to the anchor-based mean-MID of the overall,

380 personal and setting scores. However, for the domain scores the SEM exceeded the anchor-based

381 mean-MIDs, because the SDs of the domain scores were larger than the SDs of the summary

382 scores, and because the domain ICC scores were lower.

383 The known-group comparisons were based on previously reported differences in clinical

384 outcomes. Professional and setting continuity also resulted in large and relevant differences in

385 experience scores. These differences are probably due to the deviation of the expected or planned

386 process of care, which might result in a stressful event, even when this deviation is clinically

387 necessary. Differences in experiences of the other clinical known-group comparisons were not

388 relevant. It is possible that these experiences are not or only partly correlated with the clinical

389 outcomes. Another explanation might be that the experiences were reported in retrospect.

390 Perhaps women's experiences were biased afterwards by a good maternal or child outcome, by

391 better, sufficient or intensive postnatal care when complications occurred during labor. It is also

392 possible that women's experiences of the process of labor were affected by hormones and stress, 
393 or that women lacked information on what normal maternity care is. (Note that about half of the

394 women was primiparous).

395

396 Strengths

397 To our knowledge, this is the first study to clarify the meaning or relevance of score differences

398 obtained with client experience questionnaires. So far, current studies towards the MID mainly

399 focussed on quality of life scores (Brozek et al. 2006; Copay et al. 2007; Guyatt et al. 2002).

400 Secondly, the use of global ratings is debated due to their unknown validity and reliability

401 (Copay et al. 2007). By using the overarching question of the British National Patient Survey

402 Coordination Centre as anchor-question we met this critique: this global rating is extensively

403 tested and has a good content and construct validity (Graham \& Maccormick 2012). Thirdly, we

404 explored the differences between 7-8 and 8-9 changes in global rating. By doing so, we were

405 able to check the assumption that both differences were similar. Inevitably, preference scales are

406 to some extent non-linear in interpretation, which applies to both the ReproQ and to scales used

407 for anchoring. At the upper or lower ends of the scale the interpretation of gains and losses may

408 differ, and the 'degree of relevance' of one step higher (7-8) or lower (8-9) decreases. Since

409 benchmarking is usually based on the comparison of averages, the impact of non-linearity is 
410 probably small.

411

412 We previously introduced the percentage negative experiences as an alternative scoring to the

413 frequently used mean score. Three remarks should be made. Firstly, we deliberately focused on

414 the percentage negative experiences instead of percentage positive experiences. Focusing on the

415 latter may contribute to the validity of the findings. However, from a practical perspective, we

416 chose to emphasize the percentage negative experiences because in quality improvement cycles

417 most benefit can be obtained when poor performing providers or centers are identified and

418 improvements can be implemented. The percentage negative experiences seems therefore more

419 relevant for quality improvement than the percentage positive experiences. We expect that the

420 benefit of quality improvements for centers with a high percentage of positive experiences is less

421 than the benefit for poor performing centers. Secondly, both the percentage negative experiences

422 as well as the mean score can be used for benchmark purposes. Despite differences in approach,

423 both may lead to the same identification of relevant differences in subgroups (see Figure 1).

424 Finally, one could argue that our approach of the MID is conservative, as it actually defines the

425 size of a relevant minimal difference between averages on the group level on the base of

426 differences in individual global ratings. 
429 First, we sent the postnatal questionnaire six weeks after the expected date of labor, but it is

430 unknown if this timing was optimal. An invitation later than six weeks could result in recall bias

431 due to exposure to other influences (e.g. women return to work, assuming their usual habits and

432 patterns), and/or in non-response because sharing one's birth experiences may seem less

433 relevant. An invitation before six weeks is not necessarily a better option. It may result in better

434 recollection of the experiences but the risk of mood swings and hormonal disturbances might

435 affect responses and response rates.

436 Related to this: the postnatal questionnaire was not sent six weeks after the actual date of labor.

437 We only had the expected date of delivery as anchor. To protect women's privacy, we were not

438 allowed to collect the precise date of childbirth in the ReproQ. Since the expected date may

439 deviate from the true date, women may have been surveyed earlier (but not more than 2 weeks

440 earlier) when they delivered after the expected date, or about 4 to 5 weeks later for most women

441 when they delivered before the expected date. In both cases, postnatal care had already ended,

442 and it is unlikely that differences in timing of the invitation of the ReproQ may have resulted in

443 different ReproQ scores between these groups.

444 Secondly, women with a low educational level and non-Western women were underrepresented

445 despite considerable efforts to have them participate. Most likely this is selective non-response,

446 as non-Western women report more negative experiences than Western women (Scheerhagen et

447 al. 2015a), and non-Western women are more often low educated and/or more health illiterate 
448 (Agyemang et al. 2006; Engelhard 2007; Fransen et al. 2013). Addition of the non-response

449 group is likely to widen the gap between poor and good experiences. This does not necessarily

450 affect the estimated MID. Our non-Western women reported both a lower ReproQ score as well

451 as a lower global rating than Western women. Repeating the MID calculations without this

452 subgroup (non-Western women with a low educational level) resulted in about similar results.

453 When this subgroup was excluded, the percentage negative MID decreases maximum $-0.1 \%$ and

454 increases max. $+1.2 \%$. Similar, the mean MID varies -0.01 to +0.03 . Hence, the

455 underrepresentation of these subgroups has limited impact on the estimated MID. Regrettably,

456 we could not find additional evidence on the influence of selection bias on psychometric

457 properties of other client experience surveys with similar characteristics in terms of study

458 population, length and mode of administration (e.g. read out loud by clinicians vs. stand alone,

459 self-report) (Rejas et al. 2011; Vernon et al. 2010). The impact of care process, birth outcome

460 and socio-demographics on experiences scores, test-retest reliability and MID requires further

461 study.

462 Thirdly, the MID is often used to identify changes in a patient's situation over time (Brozek et al.

463 2006; Copay et al. 2007; Guyatt et al. 2002). Given the small time window of the labor phase, it

464 is unfeasible to validly assess changes in survey-based experiences within clients. Therefore, our

465 MID estimates are based on cross-sectional comparisons. Our MID cannot be used to identify

466 changes within a client, but only between health care providers, or within health care providers 
467 over time. These provider differences are more relevant than changes within clients for

468 improving the quality of maternity care by the two-stage quality cycle.

469 Finally, we aimed at suitability of the ReproQ survey across countries, by using the universal

470 WHO Responsiveness concept, by following an accepted strategy for survey development, and

471 by avoiding any preferences towards providers, specific professionals or organizational

472 structures. It is unclear if clients in other countries have the same importance ratings, experiences

473 and MIDs as Dutch clients. Other self-report instruments in maternity care, such as the

474 Women's Experience of Maternity Care Questionnaire of the NHS, overall indicate very good

475 experiences (Peterson et al. 2005; Redshaw \& Heikkila 2010; Smith 2001; Smith 2011).

476 Therefore, the MID in other developed countries will probably be in about same magnitude as

477 our MID estimates.

478

\section{Future use}

480 The psychometrics of the ReproQ appear adequate for benchmarking for targeting quality

481 improvement based on the profile of domain scores, and for monitoring of domain specific

482 quality improvements. As part of a routine two-stage quality improvement cycle, as proposed by

483 the ICHOM (ICHOM 2015), we can identify relevant differences between birth care units who

484 perform better or worse. The MID based percentage negative discriminates (known) groups

485 better than the mean-MID. Furthermore, we recommend to use a multi-item questionnaire for 
486 benchmarking, such as the ReproQ, instead of a single-item benchmark: the reliability of a

487 single-item benchmark is much lower and, unlike the ReproQ, single items are less effective in

488 guiding specific improvements.

489

490 To increase the response rate, alternative modes of data collection should be explored. One

491 suggestion is to invite women to directly fill out the questionnaire when waiting for their health

492 care professional in the waiting room. Another suggestion to minimalize selection and response

493 bias is to send all women the questionnaire including informed consent, without involvement of

494 individual health care professionals. A third suggestion is to translate and provide the

495 questionnaire in several languages for non-Western women.

496 Additionally, future use should pay attention to ethnicity and socio-economic background,

497 beyond routine case-mix adjustment procedures. Adjustment always bears the risk that

498 unintentionally worse experiences are neutralized, taking away the incentive for improvement.

499 With many benchmarking activities into place, the second part of the quality cycle urgently

500 needs more attention and explicit implementation. Evidence-based routine quality cycles are still

501 rare. Implementation requires true information-guided cycles in some detail. The benefit of such

502 an approach has been demonstrated in the evaluation of innovations (Haugum et al. 2014;

503 Weinick et al. 2014). The introduction of the MIDs in quality cycles may convince stakeholders

504 that progress through innovation is meaningful. 


\section{CONCLUSION}

507 Maternity care is continuously developing, partly based on the measurement of client

508 experiences. The ReproQ questionnaire, based on the WHO Responsiveness model, is suitable to

509 be used in quality improvement cycles: we showed good test-retest reliability, and by

510 determining the minimally important difference relevant differences can be identified. 


\section{ACKNOWLEGDEMENT}

512 We are grateful for all maternity care organizations that took part in our study. We are especially

513 grateful to Christa Cats, Hans Reinold and Ester van Dalen, who inspired the postnatal care

514 organizations to participate in our research.

515 


\section{REFERENCES}

517 Agyemang C, Denktas S, Bruijnzeels M, and Foets M. 2006. Validity of the single-item question on self-rated health status in first generation Turkish and Moroccans versus native Dutch in the Netherlands. Public Health 120:543-550.

Bland JM, and Altman DG. 1986. Statistical methods for assessing agreement between two methods of clinical measurement. Lancet 1:307-310.

Brozek JL, Guyatt GH, and Schunemann HJ. 2006. How a well-grounded minimal important difference can enhance transparency of labelling claims and improve interpretation of a patient reported outcome measure. Health Qual Life Outcomes 4:69.

Campbell SM, Roland MO, and Buetow SA. 2000. Defining quality of care. Soc Sci Med $51: 1611-1625$. 7:541-546.

CPZ. 2015. Uniform gebruik cliëntervaringslijst ReproQ in de Geboortezorg [Uniform use client experience list ReproQ in maternity care]. Available at ntervaringslijst-ReproQ-in-de-Geboortezorg2015. 
534 De Vet H, Terwee C, Mokkink L, and Knol D. 2011. Measurement in Medicine. Cambridge $535 \quad$ University Press.

536 Department of Health. 2010. Essence of Care 2010. Crown: The Stationery Office.

537 Ellis J. 2006. All inclusive benchmarking. J Nurs Manag 14:377-383.

538 Elvedi-Gasparovic V, Klepac-Pulanic T, and Peter B. 2006. Maternal and fetal outcome in 539 elective versus emergency caesarean section in a developing country. Coll Antropol $540 \quad 30: 113-118$.

541 Engelhard D. 2007. Met kennis van feiten. Vluchtelingen, nieuwkomers en gezondheid in cijfers [With knowledge of facts: figures about refugees, migrants and health]. Utrecht: Pharos.

543 Ettorchi-Tardy A, Levif M, and Michel P. 2012. Benchmarking: A method for continuous quality improvement in health. Healthcare Policy 7:e101-e119.

545 Evers AC, Brouwers HA, Hukkelhoven CW, Nikkels PG, Boon J, van Egmond-Linden A, Hillegersberg J, Snuif YS, Sterken-Hooisma S, Bruinse HW, and Kwee A. 2010. Perinatal mortality and severe morbidity in low and high risk term pregnancies in the Netherlands: prospective cohort study. BMJ 341:c5639.

Finnstrom O, Berg G, Norman A, and Otterblad Olausson P. 2006. Size of delivery unit and 550 neonatal outcome in Sweden. A catchment area analysis. Acta Obstet Gynecol Scand 85:63-67. 
552 Fransen M, Harris VC, and Essink-Bot ML. 2013. [Low health literacy in ethnic minority

553 patients: understandable language is the beginning of good healthcare]

554

555

556

557

558

559

560

561

562

563

564

565

566

567

568

569

570
Beperkte gezondheidsvaardigheden bij patienten van allochtone herkomst: alleen een tolk inzetten is meestal niet genoeg. Ned Tijdschr Geneeskd 157:A5581.

Gould JB, Qin C, and Chavez G. 2005. Time of birth and the risk of neonatal death. Obstet Gynecol 106:352-358.

Gould JB, Qin C, Marks AR, and Chavez G. 2003. Neonatal mortality in weekend vs weekday births. JAMA 289:2958-2962.

Graham C, and Maccormick S. 2012. Overarching questions for patient surveys: development report for the Care Quality Commission (CQC). Picker Institue Europe.

Guyatt GH, Osoba D, Wu AW, Wyrwich KW, Norman GR, and Clinical Significance Consensus Meeting Group. 2002. Methods to explain the clinical significance of health status measures. Mayo Clin Proc 77:371-383.

Haugum M, Danielsen K, Iversen HH, and Bjertnaes O. 2014. The use of data from national and other large-scale user experience surveys in local quality work: a systematic review. Int $J$ Qual Health Care 26:592-605.

ICHOM. 2015. How to measure. Available at http://www.ichom.org/measure/ (accessed June 26th 2015).

Kay JFL. 2007. Health care benchmarking. Hong Kong Medical Diary 12:22-27. 
571 King MT. 2011. A point of minimal important difference (MID): a critique of terminology and methods. Expert Rev Pharmacoecon Outcomes Res 11:171-184.

573 Moster D, Lie RT, and Markestad T. 1999. Relation between size of delivery unit and neonatal 574 death in low risk deliveries: population based study. Arch Dis Child Fetal Neonatal Ed 80:F221-225.

Moster D, Lie RT, and Markestad T. 2001. Neonatal mortality rates in communities with small maternity units compared with those having larger maternity units. BJOG 108:904-909.

Norman GR, Sloan JA, and Wyrwich KW. 2003. Interpretation of changes in health-related quality of life: the remarkable universality of half a standard deviation. Med Care 41:582592.

Peterson WE, Charles C, DiCenso A, and Sword W. 2005. The Newcastle Satisfaction with

Phibbs CS, Bronstein JM, Buxton E, and Phibbs RH. 1996. The effects of patient volume and level of care at the hospital of birth on neonatal mortality. Jama 276:1054-1059. hospital births. Matern Child Health J 19:764-775. 
590 Poeran J, Borsboom GJ, de Graaf JP, Birnie E, Steegers EA, Mackenbach JP, and Bonsel GJ.

5912014 . Does centralisation of acute obstetric care reduce intrapartum and first-week

592

593 mortality? An empirical study of over 1 million births in the Netherlands. Health Policy

594

Ravelli AC, Jager KJ, de Groot MH, Erwich JJ, Rijninks-van Driel GC, Tromp M, Eskes M, 595 Abu-Hanna A, and Mol BW. 2011. Travel time from home to hospital and adverse perinatal outcomes in women at term in the Netherlands. BJOG 118:457-465.

Redshaw M, and Heikkila K. 2010. Delivered with care: a national survey of women's experience of maternity care. Oxford, United Kingdom: National Perintal Epidemiology Unit.

600

Rejas J, Ruiz MA, Pardo A, and Soto J. 2011. Minimally important difference of the Treatment Satisfaction with Medicines Questionnaire (SATMED-Q). BMC Med Res Methodol

602 $11: 142$.

603

Revicki D, Hays RD, Cella D, and Sloan J. 2008. Recommended methods for determining

604 responsiveness and minimally important differences for patient-reported outcomes. J Clin

605 Epidemiol 61:102-109.

Saultz JW, and Lochner J. 2005. Interpersonal continuity of care and care outcomes: a critical review. Ann Fam Med 3:159-166. 
608 Scheerhagen M, Van Stel HF, Birnie E, Franx A, and Bonsel GJ. 2015a. Het discriminerend

609

610

611 Scheerhagen M, van Stel HF, Birnie E, Franx A, and Bonsel GJ. 2015b. Measuring Client

612

613

614

615

616

617

618

619

620

621

622

623

624

625

626

vermogen van de ReproQuestionnaire [the discriminative power of the

ReproQuestionnaire]. Rotterdam, The Netherlands: Erasmus Medical Center.

Experiences in Maternity Care under Change: Development of a Questionnaire Based on the WHO Responsiveness Model. PLoS One 10:e117031.

10.1371/journal.pone.0117031

PONE-D-14-18958 [pii]

Singh AS, Vik FN, Chinapaw MJ, Uijtdewilligen L, Verloigne M, Fernandez-Alvira JM, Stomfai

S, Manios Y, Martens M, and Brug J. 2011. Test-retest reliability and construct validity

of the ENERGY-child questionnaire on energy balance-related behaviours and their

potential determinants: the ENERGY-project. Int J Behav Nutr Phys Act 8:136.

Sitzia J, and Wood N. 1997. Patient satisfaction: a review of issues and concepts. Soc Sci Med

45:1829-1843.

Smith LF. 2001. Development of a multidimensional labour satisfaction questionnaire:

dimensions, validity, and internal reliability. Qual Health Care 10:17-22.

Smith LF. 2011. Postnatal care: development of a psychometric multidimensional satisfaction questionnaire (the WOMBPNSQ) to assess women's views. Br J Gen Pract 61:e628-637.

10.3399/bjgp11X601334 
627 Stephansson O, Dickman PW, Johansson AL, Kieler H, and Cnattingius S. 2003. Time of birth 628 and risk of intrapartum and early neonatal death. Epidemiology 14:218-222.

629 Streiner DL, and Norman GR. 2008. Health measurement scales. Oxford: University Press.

630 Tracy SK, Sullivan E, Dahlen H, Black D, Wang YA, and Tracy MB. 2006. Does size matter? A 631 population-based study of birth in lower volume maternity hospitals for low risk women.

632 BJOG 113:86-96.

Urato AC, Craigo SD, Chelmow D, and O'Brien WF. 2006. The association between time of birth and fetal injury resulting in death. Am J Obstet Gynecol 195:1521-1526.

635 Valentine NB, Bonsel GJ, and Murray CJ. 2007. Measuring quality of health care from the user's perspective in 41 countries: psychometric properties of WHO's questions on health systems responsiveness. Qual Life Res 16:1107-1125. system responsiveness: concepts, domains and measurement. In: Murray CJ, and Evans 573-596.

Vernon MK, Revicki DA, Awad AG, Dirani R, Panish J, Canuso CM, Grinspan A, Mannix S, and Kalali AH. 2010. Psychometric evaluation of the Medication Satisfaction Questionnaire (MSQ) to assess satisfaction with antipsychotic medication among schizophrenia patients. Schizophr Res 118:271-278. 
646 Weinick RM, Quigley DD, Mayer LA, and Sellers CD. 2014. Use of CAHPS patient experience

647 surveys to assess the impact of health care innovations. Jt Comm J Qual Patient Saf

648 40:418-427.

649 Wensing M, Jung HP, Mainz J, Olesen F, and Grol R. 1998. A systematic review of the literature 650 on patient priorities for general practice care. Part 1: Description of the research domain. Soc Sci Med 47:1573-1588.

652

Williams B. 1994. Patient satisfaction: a valid concept? Soc Sci Med 38:509-516. 


\section{Table 1 (on next page)}

Table 1. Description of the eight WHO Responsiveness domains. 
1 TABLES

2

3 Table 1. Description of the eight WHO Responsiveness domains.

4
Domain
Description
Dignity
Receiving care in a respectful, caring, non-discriminatory setting.
Autonomy
The need to involve the individuals in the decision-making process to the extent that they wish this to occur; the right of patients of sound mind to refuse treatment for themselves.
Confidentiality The privacy of the environment in which consultations are conducted by health providers; the confidentiality of medical records and information about individuals.
Communication The notion that providers explain clearly to the patient and family The nature of the illness, and details for the required treatment and options. It also includes providing time for patients to understand their symptoms and to ask questions
Prompt attention Care provided readily or as soon as necessary
Social The feeling of being cared for and loved, valued, esteemed and able considerations to count on others should the need arise.
Basic Amenities The extent to which the physical infrastructure of a health facility is welcoming and pleasant
Choice and The power or opportunity to select, which requires more than one continuity option. 


\section{Table 2 (on next page)}

Table 2. Characteristics of women who filled out the test $(n=4675)$ and the retest $(n=208)^{\$}$.

${ }^{\$}$ The percentage of missing data was below $5 \%$ in all characteristics, and will therefore not be presented.

* Significant difference between the participating women of the test and women participating the retest. 
2

\section{TABLES}

Table 2. Characteristics of women who filled out the test $(n=4675)$ and the retest $(n=208)^{\$}$.

\begin{tabular}{|c|c|c|c|}
\hline & & Test (\%) & $\begin{array}{r}\text { Retest } \\
(\%)\end{array}$ \\
\hline \multicolumn{4}{|l|}{ Socio-demographics } \\
\hline \multirow[t]{4}{*}{ Age } & $\leq 24$ years & 6 & 4 \\
\hline & $25-29$ years & 30 & 31 \\
\hline & $30-34$ years & 42 & 48 \\
\hline & $\geq 35$ years & 22 & 17 \\
\hline Parity & Primiparous & 52 & 48 \\
\hline Ethnic background* & Non-Western & 9 & 3 \\
\hline \multirow[t]{3}{*}{ Educational level } & Low & 8 & 5 \\
\hline & Middle & 35 & 33 \\
\hline & High & 57 & 61 \\
\hline \multirow[t]{3}{*}{ Marital status } & Married/living together & 96 & 97 \\
\hline & Relationship, not living together & 2 & 2 \\
\hline & No relationship & 2 & 2 \\
\hline \multicolumn{4}{|l|}{ Care } \\
\hline \multirow{5}{*}{$\begin{array}{l}\text { Professional continuity } \\
\text { Setting continuity }\end{array}$} & No & 48 & 51 \\
\hline & Primary care only & 37 & 34 \\
\hline & Secondary care only & 16 & 15 \\
\hline & $\begin{array}{l}\text { Referral to secondary care } \\
\text { during pregnancy }\end{array}$ & 11 & 17 \\
\hline & $\begin{array}{l}\text { Referral to secondary care } \\
\text { during parturition }\end{array}$ & 36 & 34 \\
\hline Onset of delivery & Outside office hours & 70 & 64 \\
\hline \multirow[t]{3}{*}{ Travel time* } & None or by choice & 70 & 79 \\
\hline & $<15$ minutes during delivery & 18 & 14 \\
\hline & $\geq 15$ minutes during delivery & 12 & 7 \\
\hline \multirow[t]{3}{*}{ Cesarean section } & No & 87 & 87 \\
\hline & Planned cesarean & 4 & 3 \\
\hline & Emergency cesarean & 8 & 10 \\
\hline \multirow{4}{*}{$\begin{array}{l}\text { Hospital size of the } \\
\text { perinatal unit }\end{array}$} & $<750$ deliveries per year & & \\
\hline & & 12 & 11 \\
\hline & 750-1499 deliveries per year & 47 & 44 \\
\hline & $\geq 1500$ deliveries per year & 40 & 46 \\
\hline \multicolumn{4}{|l|}{ Quality } \\
\hline \multirow[t]{5}{*}{ Picker overall rating* } & $\leq 6$ & 8 & 4 \\
\hline & 7 & 16 & 8 \\
\hline & 8 & 34 & 39 \\
\hline & 9 & 26 & 30 \\
\hline & 10 & 16 & 18 \\
\hline
\end{tabular}

$\$$ The percentage of missing data was below $5 \%$ in all characteristics, and will therefore not be presented. 
6 * Significant difference between the participating women of the test and women participating the retest. 


\section{Table 3(on next page)}

Table 3. Test-retest reliability of the experience during labor, on percentage women with a negative experience and mean score $(n=208)$.

* Most negative experience (never) in a domain and/or 'sometimes' in the individually chosen 2 most important domains. ^ The Bland Altman plot of the total score are presented in supplementary file 2. 
1 Table 3. Test-retest reliability of the experience during labor, on percentage women with a negative experience and mean score $(n=208)$.

\begin{tabular}{|c|c|c|c|c|c|c|c|c|}
\hline \multirow[t]{2}{*}{ Score } & \multicolumn{3}{|c|}{ Negative experience $^{*}$} & \multicolumn{5}{|c|}{ Mean experience } \\
\hline & Test (\%) & Retest (\%) & $\begin{array}{l}\text { Absolute } \\
\text { agreement (\%) }\end{array}$ & $\begin{array}{l}\text { Test mean } \\
\text { (SD) }\end{array}$ & $\begin{array}{l}\text { Retest } \\
\text { mean (SD) }\end{array}$ & $\mathrm{ICC}$ & Bias & $\begin{array}{l}\text { Limits of } \\
\text { agreement^ }\end{array}$ \\
\hline Overall & $46.6 \%$ & $39.9 \%$ & $78.8 \%$ & $3.79(0.21)$ & $3.78(0.23)$ & 0.78 & 0.01 & 0.31 \\
\hline Personal domains & $31.7 \%$ & $27.4 \%$ & $82.2 \%$ & $3.75(0.27)$ & $3.74(0.28)$ & 0.74 & 0.02 & 0.42 \\
\hline Setting domains & $25.0 \%$ & $22.6 \%$ & $83.2 \%$ & $3.82(0.21)$ & $3.83(0.22)$ & 0.74 & -0.01 & 0.31 \\
\hline Dignity & $3.4 \%$ & $2.9 \%$ & $94.7 \%$ & $3.89(0.23)$ & $3.84(0.27)$ & 0.62 & 0.05 & 0.48 \\
\hline Autonomy & $27.9 \%$ & $26.0 \%$ & $86.5 \%$ & $3.46(0.59)$ & $3.50(0.50)$ & 0.65 & -0.04 & 0.88 \\
\hline Confidentiality & $1.9 \%$ & $1.4 \%$ & $96.6 \%$ & $3.84(0.36)$ & $3.81(0.35)$ & 0.49 & 0.02 & 0.74 \\
\hline Communication & $2.4 \%$ & $1.4 \%$ & $98.1 \%$ & $3.81(0.34)$ & $3.79(0.34)$ & 0.70 & 0.02 & 0.55 \\
\hline Prompt Attention & $6.7 \%$ & $4.8 \%$ & $94.2 \%$ & $3.81(0.30)$ & $3.82(0.29)$ & 0.64 & -0.01 & 0.49 \\
\hline Social Considerations & $2.4 \%$ & $1.9 \%$ & $98.6 \%$ & $3.89(0.28)$ & $3.90(0.24)$ & 0.54 & -0.01 & 0.49 \\
\hline Basic Amenities & $1.4 \%$ & $1.0 \%$ & $99.5 \%$ & $3.89(0.25)$ & $3.89(0.27)$ & 0.58 & 0.05 & 0.48 \\
\hline Choice and Continuity & $18.3 \%$ & $18.3 \%$ & $85.6 \%$ & $3.69(0.44)$ & $3.69(0.45)$ & 0.62 & 0.00 & 0.78 \\
\hline
\end{tabular}

2

$3{ }^{*}$ Most negative experience (never) in a domain and/or 'sometimes' in the individually chosen 2 most important domains.

$\wedge$ The Bland Altman plot of the total score are presented in supplementary file 2. 


\section{Table 4 (on next page)}

Table 4: Minimally important difference of the experience during labor based on the mean scores and the percentage women that had a negative experience $(n=3841)$

\$ Due to a software problem this item was not presented to $20 \%$ of the participating women.

* Most negative experience (never) in an domain and/or 'sometimes' in the individually chosen 2 most important domains. 
1 Table 4: Minimally important difference of the experience during labor based on the mean scores

2 and the percentage women that had a negative experience $(n=3841)^{\$}$

3

\begin{tabular}{|c|c|c|c|c|c|c|c|c|}
\hline \multirow{3}{*}{ Global rating per score } & \multirow{3}{*}{$\mathrm{N}$} & \multicolumn{2}{|c|}{$\begin{array}{c}\text { Negative } \\
\text { experience* }\end{array}$} & \multicolumn{5}{|c|}{ Mean experience } \\
\hline & & \multirow[t]{2}{*}{$\% \mathrm{Neg}$} & \multirow{2}{*}{$\begin{array}{l}\text { Anchor } \\
\text { based } \\
\text { MID }\end{array}$} & \multirow[t]{2}{*}{ Mean } & \multirow{2}{*}{$\begin{array}{l}\text { Anchor } \\
\text { based } \\
\text { MID }\end{array}$} & \multirow[t]{2}{*}{ SD } & \multicolumn{2}{|c|}{$\begin{array}{l}\text { Distribution- } \\
\text { based MID }\end{array}$} \\
\hline & & & & & & & SEM & $1 / 2 \mathrm{SD}$ \\
\hline \multicolumn{9}{|l|}{ Total score } \\
\hline $7(\rightarrow 8)$ & 584 & $60,4 \%$ & $11,0 \%$ & 3,59 & 0,15 & & & \\
\hline 8 (ref) & 1322 & $49,4 \%$ & & 3,74 & & 0,29 & 0,14 & 0,14 \\
\hline $9(\leftarrow 8)$ & 1021 & $40,2 \%$ & $9,2 \%$ & 3,84 & 0,10 & & & \\
\hline \multicolumn{9}{|l|}{ Personal score } \\
\hline $7(\rightarrow 8)$ & 584 & $44,9 \%$ & $8,5 \%$ & 3,52 & 0,17 & & & \\
\hline 8 (ref) & 1322 & $36,4 \%$ & & 3,69 & & 0,35 & 0,18 & 0,17 \\
\hline $9(\leftarrow 8)$ & 1021 & $27,5 \%$ & $8,9 \%$ & 3,80 & 0,11 & & & \\
\hline \multicolumn{9}{|l|}{ Setting score } \\
\hline $7(\rightarrow 8)$ & 584 & $36,6 \%$ & $11,6 \%$ & 3,66 & 0,13 & & & \\
\hline 8 (ref) & 1322 & $25,0 \%$ & & 3,79 & & 0,28 & 0,14 & 0,14 \\
\hline $9(\leftarrow 8)$ & 1021 & $18,8 \%$ & $6,2 \%$ & 3,87 & 0,08 & & & \\
\hline \multicolumn{9}{|l|}{ Dignity } \\
\hline $7(\rightarrow 8)$ & 584 & $11,6 \%$ & $6,9 \%$ & 3,66 & 0,18 & & & \\
\hline 8 (ref) & 1322 & $4,7 \%$ & & 3,84 & & 0,34 & 0,21 & 0,17 \\
\hline $9(\leftarrow 8)$ & 1021 & $2,0 \%$ & $2,7 \%$ & 3,93 & 0,09 & & & \\
\hline \multicolumn{9}{|l|}{ Autonomy } \\
\hline $7(\rightarrow 8)$ & 584 & $36,0 \%$ & $5,4 \%$ & 3,22 & 0,17 & & & \\
\hline 8 (ref) & 1322 & $30,6 \%$ & & 3,39 & & 0,58 & 0,35 & 0,29 \\
\hline $9(\leftarrow 8)$ & 1021 & $25,6 \%$ & $5,0 \%$ & 3,56 & 0,17 & & & \\
\hline \multicolumn{9}{|l|}{ Confidentiality } \\
\hline $7(\rightarrow 8)$ & 584 & $5,7 \%$ & $2,0 \%$ & 3,64 & 0,17 & & & \\
\hline 8 (ref) & 1322 & $3,7 \%$ & & 3,80 & & 0,46 & 0,33 & 0,23 \\
\hline $9(\leftarrow 8)$ & 1021 & $1,8 \%$ & $1,9 \%$ & 3,88 & 0,08 & & & \\
\hline \multicolumn{9}{|l|}{ Communication } \\
\hline $7(\rightarrow 8)$ & 584 & $5,3 \%$ & $2,4 \%$ & 3,55 & 0,17 & & & \\
\hline 8 (ref) & 1322 & $2,9 \%$ & & 3,73 & & 0,40 & 0,22 & 0,20 \\
\hline $9(\leftarrow 8)$ & 1021 & $0,7 \%$ & $2,2 \%$ & 3,84 & 0,11 & & & \\
\hline \multicolumn{9}{|l|}{ Prompt Attention } \\
\hline $7(\rightarrow 8)$ & 584 & $10,3 \%$ & $4,4 \%$ & 3,62 & 0,13 & & & \\
\hline
\end{tabular}




\begin{tabular}{|c|c|c|c|c|c|c|c|c|}
\hline 8 (ref) & 1322 & $5,9 \%$ & & 3,75 & & 0,35 & 0,35 & 0,18 \\
\hline $9(\leftarrow 8)$ & 1021 & $3,9 \%$ & $2,0 \%$ & 3,85 & 0,10 & & & \\
\hline \multicolumn{9}{|c|}{ Social Considerations } \\
\hline $7(\rightarrow 8)$ & 584 & $3,3 \%$ & $1,0 \%$ & 3,75 & 0,12 & & & \\
\hline 8 (ref) & 1322 & $2,3 \%$ & & 3,87 & & 0,33 & 0,22 & 0,16 \\
\hline $9(\leftarrow 8)$ & 1021 & $1,5 \%$ & $0,8 \%$ & 3,91 & 0,04 & & & \\
\hline \multicolumn{9}{|c|}{ Basic Amenities } \\
\hline $7(\rightarrow 8)$ & 584 & $4,6 \%$ & $2,5 \%$ & 3,80 & 0,07 & & & \\
\hline 8 (ref) & 1322 & $2,1 \%$ & & 3,87 & & 0,30 & 0,20 & 0,15 \\
\hline $9(\leftarrow 8)$ & 1021 & $1,6 \%$ & $0,5 \%$ & 3,92 & 0,05 & & & \\
\hline \multicolumn{9}{|c|}{ Choice and Continuity } \\
\hline $7(\rightarrow 8)$ & 584 & $26,2 \%$ & $8,0 \%$ & 3,46 & 0,19 & & & \\
\hline 8 (ref) & 1322 & $18,2 \%$ & & 3,65 & & 0,52 & 0,32 & 0,26 \\
\hline $9(\leftarrow 8)$ & 1021 & $13,3 \%$ & $4,9 \%$ & 3,78 & 0,13 & & & \\
\hline
\end{tabular}

4 \$ Due to a software problem this item was not presented to $20 \%$ of the participating women.

5 * Most negative experience (never) in an domain and/or 'sometimes' in the individually chosen 2 most important domains. 


\section{1}

Difference in ReproQ in terms of negative score (A) and mean score (B), between the least preferred and the most preferred state, in 6 known-groups $(n=4883)$.

Professional continuity - difference supervisor of the delivery is known vs. unknown (52\% / 48\%)

Setting continuity - difference primary care only vs. referred during labor (37\% / 36\%)

Onset of delivery - difference in vs. outside office hours (30\% / 70\%)

Travel time - difference women had to travel $<15$ minutes vs. $\geq 15$ minutes, when transferred from home to hospital during labor (17\% / 11\%).

Cesarean section - difference planned vs. emergency cesarean section (4\% / 8\%)

Hospital size - difference $<750$ deliveries per year vs. $\geq 1500$ deliveries per year ( $3 \%$ / 12\%). 
A)

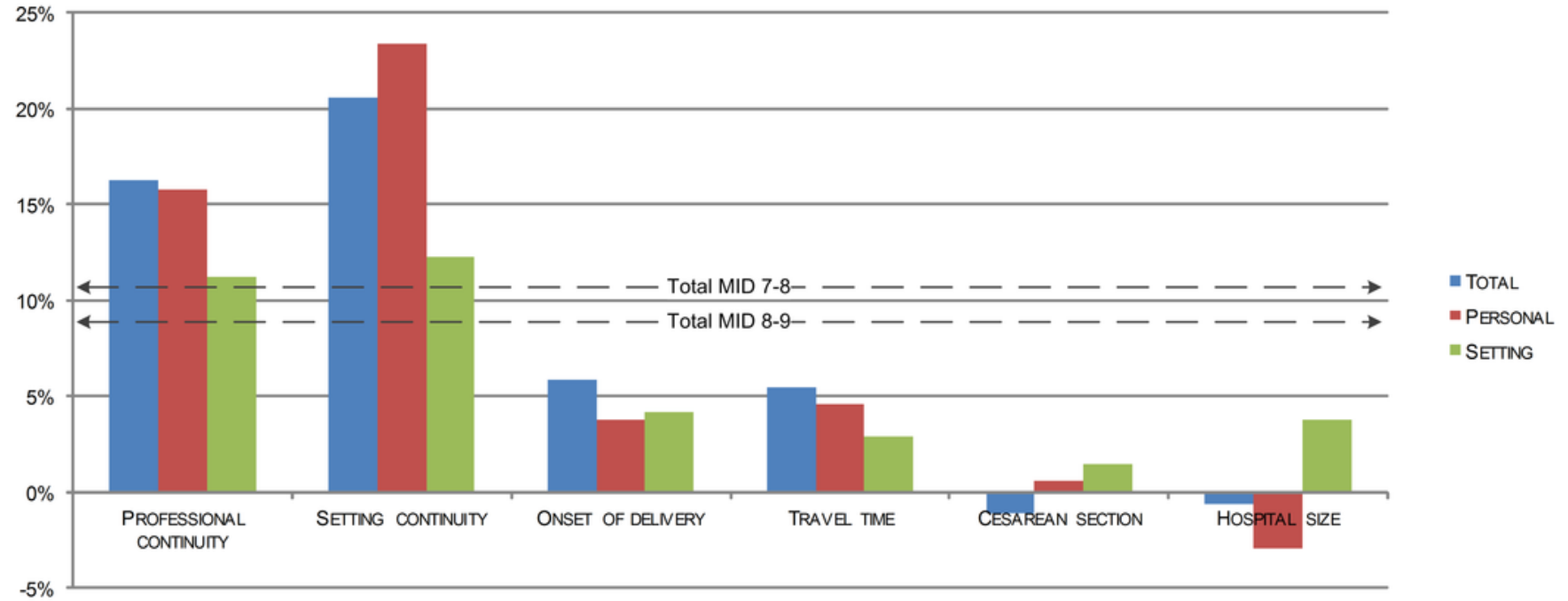

B) 0

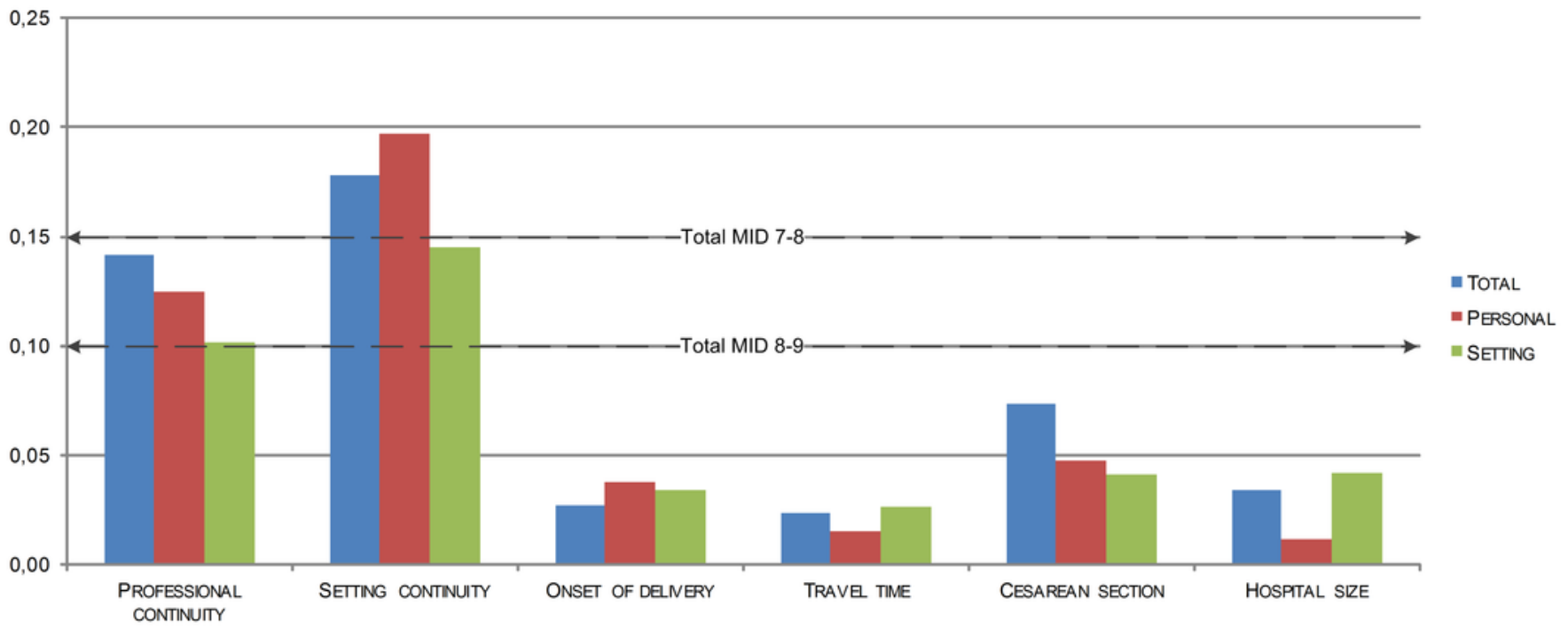

